INFLAMMATORY BOWEL DISEASE

\title{
Genetic basis for increased intestinal permeability in families with Crohn's disease: role of CARD15 3020insC mutation?
}

\author{
S Buhner, C Buning, J Genschel, K Kling, D Herrmann, A Dignass, I Kuechler, \\ S Krueger, H H-J Schmidt, H Lochs
}

See end of article for authors' affiliations

Correspondence to: Dr S Bühner, Department of Gastroenterology, Hepatology, and Endocrinology, Campus Charité Mitte, CharitéUniversitätsmedizin Berlin, Schumannstr 20-21, 10117 Berlin, Germany; sabine.buehner@ charite.de

Revised version received 16 June 2005

Accepted for publication

21 June 2005

Published online first

29 July 2005
Background and aim: A genetically impaired intestinal barrier function has long been suspected to be a predisposing factor for Crohn's disease (CD). Recently, mutations of the capsase recruitment domain family, member 15 (CARD15) gene have been identified and associated with CD. We hypothesise that a CARD 15 mutation may be associated with an impaired intestinal barrier.

Methods: We studied 128 patients with quiescent CD, 129 first degree relatives (CD-R), 66 non-related household members (CD-NR), and 96 healthy controls. The three most common CARD15 polymorphisms (R702W, G908R, and 3020insC) were analysed and intestinal permeability was determined by the lactulose/mannitol ratio.

Results: Intestinal permeability was significantly increased in CD and CD-R groups compared with CD-NR and controls. Values above the normal range were seen in $44 \%$ of $C D$ and $26 \%$ of CD-R but only in $6 \%$ of CD-NR, and in none of the controls. A household community with CD patients, representing a common environment, was not associated with increased intestinal permeability in family members. However, 40\% of CD first degree relatives carrying a CARD15 3020insC mutation and 75\% (3/4) of those CD-R with combined 3020insC and R702W mutations had increased intestinal permeability compared with only $15 \%$ of wild-types, indicating a genetic influence on barrier function. R702W and G908R mutations were not associated with high permeability.

Conclusions: In healthy first degree relatives, high mucosal permeability is associated with the presence of a CARD 15 3020insC mutation. This indicates that genetic factors may be involved in impairment of intestinal barrier function in families with IBD.
$\mathrm{T}$ he integrity of gut barrier function in patients with inflammatory bowel diseases (IBD) is known to be impaired. ${ }^{1-8}$ However, the causes and consequences of the "leaky gut" in IBD are still under discussion. The leading concept assumes that barrier dysfunction reflects a very early defect in the disease process that might be genetically driven and triggered by environmental factors such as luminal antigens or bacteria. ${ }^{9}{ }^{10}$ By bypassing normal antigen uptake in a situation of high permeability, induction of a physiological immune response is altered. An unsuppressed immune response is induced, initiating disease or worsening disease outcome.

To establish the role of permeability in the causality of Crohn's disease (CD), family studies have been performed. In a number of studies approximately $10-54 \%$ of first degree relatives of $\mathrm{CD}$ patients showed increased intestinal permeability in the absence of clinical symptoms. ${ }^{367}$ Although only a subgroup of relatives had increased values, data support the genetic concept. This is in line with a case report revealing changes in intestinal permeability eight years before the onset of CD. ${ }^{11}$ Interestingly, three studies showed a high prevalence of increased intestinal permeability in spouses of CD patients, ${ }^{78}{ }^{12}$ suggesting that environmental rather than genetic factors may play a role.

Recently, mutations of the capsase recruitment domain family, member 15/nucleotide binding oligomerisation domain 2 (CARD15/NOD2) gene have been identified and associated with CD. ${ }^{13-15}$ To date, the relationship between IBD and the CARD15 mutation has been confirmed by many authors. ${ }^{16-19}$ The CARD15 protein is a member of the Ced-4 superfamily of apoptosis regulators with homology to the plant disease resistance gene products being implicated in the recognition of pathogen components. ${ }^{13}{ }^{14}$ CARD15 protein acts as an intracellular receptor for the bacterial cell wall component peptidoglycan through the $\mathrm{C}$ terminal leucine rich repeats (LRRs). The bioactive component of peptidoglycan is muramyl dipeptide. ${ }^{20}{ }^{21}$ This interaction leads to activation of nuclear transcription factor $\kappa \mathrm{B}(\mathrm{NF} \kappa \mathrm{B})$ which plays a central role in innate immunity. ${ }^{20}$ The three most common genetic variants in CARD15 are the two missense mutations Arg702Trp (R702W) and Gly908Arg (G908R) and the insertion frameshift mutation at nucleotide 3020 (3020insC), the latter leading to truncation of the $C$ terminal 33 amino acids in the LRR region. This frameshift mutation is associated with hyporesponsiveness to peptidoglycan and attenuated NFKB activation. ${ }^{18} 20$ Epithelial cells transfected with the CARD15 3020insC mutant overexpressed the CARD15/NOD2 protein but displayed a diminished antibacterial defence function. ${ }^{22}$

With this in mind, the question arises of whether CARD15 mutations or environmental factors are associated with gastrointestinal barrier dysfunction. Therefore, we analysed

Abbreviations: CARD15/NOD2, capsase recruitment domain family, member 15/nucleotide binding oligomerisation domain 2; CD, Crohn's disease; $C D-R$, first degree relative of a patient with $C D ; C D-N R$, nonrelated household member living with a patient with $C D$; IBD,

inflammatory bowel disease; LRR, $C$ terminal leucine rich repeats; $N F \kappa B$, nuclear transcription factor $\mathrm{\kappa B}$; TNF- $\alpha$, tumour necrosis factor $\alpha$; NSAID non-steroidal anti-inflammatory drugs; $\mathrm{PI}$, permeability index; WT, wildtype 
intestinal permeability and the three most common CARD15 polymorphisms (R702W, G908R, and 3020insC) in patients with CD, their first degree relatives, and non-related household members. Strikingly, intestinal permeability in phenotypically normal first degree relatives of $\mathrm{CD}$ patients was increased compared with controls and significantly associated with the CARD15 3020insC mutation.

\section{METHODS \\ Participants \\ Patients}

A total of 128 patients with a confirmed diagnosis of CD were included in the present study ( 85 females, 43 males; median age 36 years (range 16-63)). Patients were recruited from Charité University Hospital (Berlin, Germany). Diagnosis of CD was based on standard clinical, radiological, endoscopic, and histological criteria. The disease was located in the terminal ileum in 37 patients $(30 \%)$, the colon in 20 patients $(16 \%)$, the ileocolon in 53 patients $(40 \%)$, and the upper gastrointestinal tract in 18 patients $(14 \%)$. Ninety patients were currently taking mesalamine, 31 oral prednisolone ( $\mathrm{n}=19 \leqslant 10 \mathrm{mg}$ ), 19 oral budenoside, five sulfasalazopyridine containing compounds, and 21 patients had no current medication. All patients were in clinical remission; mean CD activity index was 82 (range 5-148). None of the patients was taking antibiotics or tumour necrosis factor $\alpha$ (TNF- $\alpha)$ antibodies.

First degree relatives (CD-R)

A total of 129 first degree relatives of patients with CD were studied ( 83 females, 46 males; median age 43 years (range 15-82)), including 62 parents, 27 siblings, and 40 children of $\mathrm{CD}$ patients. Forty eight of the CD-R had been living in the same household with CD patients, at least since the time of diagnosis (CD-R household members).

Non-relatives (CD-NR)

Sixty six non-blood relatives of CD patients were included in the present study ( 23 females, 43 males; median age 38 years (range 20-68)). Except for one person (the stepfather of a CD patient), all CD-NR were the partners of the patients. All lived in the same household with the patient, the majority $(\mathrm{n}=5 \mathrm{l})$ since the time of $\mathrm{CD}$ diagnosis.

\section{Healthy controls}

A total of 96 healthy volunteers (56 females, 40 males; median age 32 years (range 19-65)) were used as controls. They were predominantly recruited in cooperation with the Department of Transfusion Medicine of Charité University Hospital.

\section{Predetermined exclusion criteria}

Predetermined exclusion criteria were any (for CD patients: concomitant) gastrointestinal or hepatobiliary disease, severe neurological, endocrine, cardiovascular, pulmonary, or renal disease, gastrectomy, colectomy, or extensive resection of the small bowel, cancer, rheumatoid based diseases, acute infections, acute urticaria, or pregnancy. Alcohol and nonsteroidal anti-inflammatory drugs (NSAID) were forbidden for at least 48 hours before the test. CD family members (CD$\mathrm{R}$ and $\mathrm{CD}-\mathrm{NR}$ ) and controls showing any signs or symptoms of IBD-like recurrent diarrhoea, flatulence, gastrointestinal pain, or blood in the stool, or any other chronic illness, were excluded from the study. Additionally, drug intake, including glucocorticosteroids, antimicrobial agents, immunosuppressives, and regular consumption of NSAIDs (for example, for arthropathies) was not allowed for family members or controls. Persons with any previous gastrointestinal or colorectal surgery were excluded. In general, no drugs influencing gut functions, including laxatives and antidiarrhoeal agents, were allowed for 24 hours before the onset of the test. Finally, smoking was strictly forbidden in the morning before the test and during the test. Moreover, every participant was asked to record alcohol and cigarette consumption and medicine intake for one week before the test.

\section{Study regimen}

The intestinal permeability test was carried out at home by all persons. Blood samples for genetic analysis were taken in a subgroup of study participants, either at the outpatient department of the clinical centres involved or at their family doctors. A standardised questionnaire was used for data inquiry.

\section{Intestinal permeability}

Intestinal permeability was assessed using a sugar drink test, as previously described in detail. ${ }^{23}$ The test is based in principle on measurement of urinary excretion of an orally administered non-metabolised sugar probe molecules; the lactulose/mannitol ratio (permeability index, PI) served as a marker for intestinal permeability.

After an overnight fast, each subject provided a pre-test urine sample. Then they drank a solution containing $10 \mathrm{~g}$ lactulose and $5 \mathrm{~g}$ mannitol dissolved in $100 \mathrm{ml}$ water. Urine was collected over five hours with sodium azide as preservative. Subjects went without food during the test but were allowed to drink water after two hours. Total urine volume was recorded on completion of the test and a $10 \mathrm{ml}$ aliquot was stored at $-20^{\circ} \mathrm{C}$ until analysis.

For sample preparation the protein was removed with sulfosalicilic acid and urine was desalted with Amberlite MB3 resin in acetate form. Using meso-erythritol and turanose as internal standards, the sugars were separated, analysed, and quantified by high performance liquid chromatography with pulsed electrochemical detection (Dionex, Idstein, Germany); chromatography module $250 \times 40 \mathrm{~mm}$ Carbopac

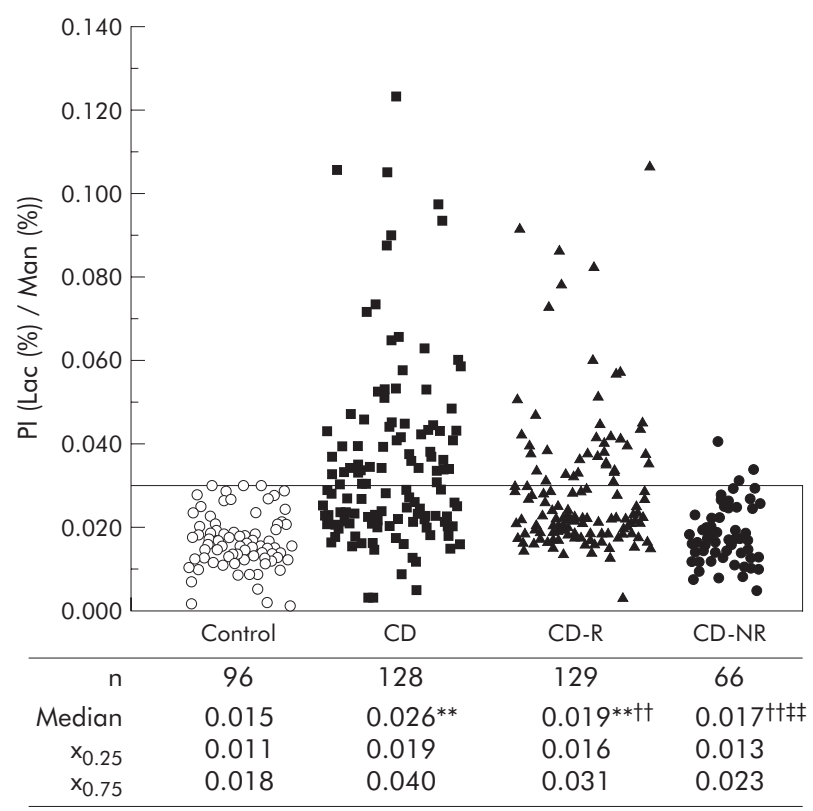

Figure 1 Intestinal permeability in healthy controls, in patients with Crohn's disease (CD), their first degree relatives (CD-R), and in nonblood relatives (CD-NR) (median (interquartile range: 25th-75th percentile)). Intestinal permeability was increased in $C D$ and $C D-R$ compared with controls. PI, permeability index (lactulose (Lac)/mannitol (Man) ratio). $p<0.004$ compared with: ** controls, $†+C D, ~ \neq \neq C D-R$ (Mann-Whitney test). Bordered area range of normal permeability. 
Table 1 Intestinal permeability in subgroups of first degree relatives of patients with Crohn's disease (CD-R)

\begin{tabular}{llll}
\hline & $\mathbf{n}$ & PI (Lac\%/Man\%)* & $\begin{array}{l}\text { Subjects with high } \\
\text { permeability }\end{array}$ \\
\hline CD-R (household members) & 48 & $0.019(0.015-0.030)$ & $25 \%(12)$ \\
CD-R (non-household members) & 61 & $0.020(0.017-0.034)$ & $31 \%(19)$ \\
\hline *Values are median (interquartile range). & & \\
PI, permeability index (lactulose (Lac)/mannitol (Man) ratio). & \\
\hline
\end{tabular}

PA-l column (Dionex); eluent $150 \mathrm{mmol} \mathrm{NaOH}$; flow $1 \mathrm{ml} / \mathrm{min}$. Results were expressed as percentage recovery of the ingested dose of the sugars.

\section{Gene analysis}

All methods used for gene analysis have been described previously in detail. ${ }^{19}$ Briefly, genomic DNA was prepared from peripheral blood using commercially available extraction columns (QIAmp Blood Kit; Qiagen, Hilden, Germany). After amplification of exon 11 of the CARD15 gene and cycle sequencing using an ABI 310 automatic sequencer (Applied Biosystems, Weiterstadt, Germany), genotyping of the three CARD15 polymorphisms (R702W, G908R, and 3020insC) was performed using the fluorogenic $5^{\prime}$-nuclease assay. Primers and probes were the same as previously described. ${ }^{19}$

\section{Statistics}

Differences in permeability data between the study groups were analysed using the Kruskal-Wallis test and, in case of significance, the Mann-Whitney $U$ test. The level of significance $(\alpha)$ was chosen at $5 \%$. An $\alpha$ adjustment was applied by Bonferroni procedure. Differences between incidences were tested by cross tables and $\chi^{2}$ statistics.

The upper limit of normal intestinal permeability was defined as mean value $+2 \mathrm{SD}$ of the control group $(=0.03)$, as previously described. ${ }^{523}$

\section{Ethics}

The study was approved by the ethics commission of the Charité University Hospital and informed consent was obtained from each participant.

\section{RESULTS}

\section{Intestinal permeability}

Intestinal permeability was significantly increased in CD patients and in CD-R compared with controls and CD-NR (fig 1). Values above the normal range were seen in $44 \%$ of CD patients and in $26 \%$ of CD-R, but in only $6 \%$ of CD-NR and in none of the controls. This already refutes the role of an environmental factor being predominantly responsible for barrier dysfunction. To further differentiate between genetic and environmental factors, we concentrated on the group of CD first degree relatives and used the household community with the $\mathrm{CD}$ patients to investigate the influence of environmental factors on intestinal permeability. Thirty seven per cent $(n=48)$ of CD-R lived in the same household with the patient since the time of $\mathrm{CD}$ diagnosis (CD-R household members). Forty seven per cent $(n=61)$ of CD-R lived in a different household than the patient, both at the time of CD diagnosis and at the time of the test (CD-R nonhousehold members). As seen in table 1, intestinal permeability did not differ between these two groups, indicating that household community, as an environmental factor, does not explain the general differences in intestinal barrier function described above.

We further analysed the effect of smoking habits in each group. The proportion of smokers showing a PI above the normal limit was higher compared with non-smokers $(61 \% v$ $37 \% ; p=0.019$, Fisher's exact test) in CD patients but in none of the other groups (CD-R, p=0.602; CD-NR, p=0.563). Median values for intestinal permeability did not differ between smokers and non-smokers in any of the groups. Additionally, a slight gender disproportion in the CD-R and

\begin{tabular}{|c|c|c|c|c|}
\hline Genotype & Control & $C D$ & CD-R & CD-NR \\
\hline $\begin{array}{l}\text { 3020insC } \\
\text { Wild-type } \\
\text { Heterozygous mutant } \\
\text { Homozygous mutant }\end{array}$ & $\begin{array}{l}(n=62) \\
98 \%(61) \\
2 \%(1)\end{array}$ & $\begin{array}{l}(n=121) \\
78 \%(94) \\
18 \%(22) \\
4 \%(5) \\
p<0.0005\end{array}$ & $\begin{array}{l}(n=106) \\
77 \%(82) \\
22 \%(23) \\
1 \%(1) \\
p<0.0005\end{array}$ & $\begin{array}{l}(n=50) \\
88 \%(44) \\
10 \%(5) \\
2 \%(1) \\
p=0.044\end{array}$ \\
\hline $\begin{array}{l}\text { R702W } \\
\text { Wild-type } \\
\text { Heterozygous mutant } \\
\text { Homozygous mutant }\end{array}$ & $\begin{array}{l}(n=62) \\
94 \%(58) \\
6 \%(4)\end{array}$ & $\begin{array}{l}(n=120) \\
83 \%(100) \\
14 \%(17) \\
3 \%(3) \\
p=0.06\end{array}$ & $\begin{array}{l}(n=106) \\
84 \%(89) \\
15 \%(16) \\
1 \%(1) \\
p=0.091\end{array}$ & $\begin{array}{l}(n=50) \\
88 \%(44) \\
12 \%(6) \\
p=0.337\end{array}$ \\
\hline $\begin{array}{l}\text { G908R } \\
\text { Wild-type } \\
\text { Heterozygous mutant }\end{array}$ & $\begin{array}{l}(n=62) \\
97 \%(60) \\
3 \%(2)\end{array}$ & $\begin{array}{l}(n=120) \\
92 \%(110) \\
8 \%(10) \\
p=0.343\end{array}$ & $\begin{array}{l}(n=105) \\
98 \%(103) \\
2 \%(2) \\
p=0.628\end{array}$ & $\begin{array}{l}(n=50) \\
98 \%(49) \\
2 \%(1) \\
p=1.000\end{array}$ \\
\hline Overall & $\begin{array}{l}(n=62) \\
11 \%(7)\end{array}$ & $\begin{array}{l}(n=121) \\
43 \%(52)\end{array}$ & $\begin{array}{l}(n=106) \\
37 \%(39)\end{array}$ & $\begin{array}{l}(n=50) \\
24 \%(12)\end{array}$ \\
\hline \multicolumn{5}{|c|}{$\begin{array}{l}C D \text {, Crohn's disease; } C D-R \text {, first degree relative of a patient with CD; CD-NR, non-related household member } \\
\text { living with a patient with CD. } \\
\text { Overall, number of persons carrying at least one mutation. } \\
\text { p values for differences in frequency distribution of CARD } 15 \text { mutations between CD groups and controls (Fisher's } \\
\text { exact test) } \\
\text { Number of cases in parentheses. }\end{array}$} \\
\hline
\end{tabular}


Table 3 Frequencies of normal and high intestinal permeability with respect to the occurrence of the three CARD15 mutations (3020ins C, R702W, and G908R)

\begin{tabular}{|c|c|c|c|c|c|c|c|c|}
\hline & PI & WT & 3020 ins C & R702W & G908R & $\begin{array}{l}\text { 3020insC } \\
\text { and } R 702 W\end{array}$ & $\begin{array}{l}\text { 3020insC } \\
\text { and G908R }\end{array}$ & $\begin{array}{l}R 702 W \text { and } \\
\text { G908R }\end{array}$ \\
\hline \multirow[t]{3}{*}{$C D$} & & $\mathrm{n}=68$ & $\mathrm{n}=23$ & $\mathrm{n}=16$ & $\mathrm{n}=8$ & $\mathrm{n}=3$ & $\mathrm{n}=1$ & $\mathrm{n}=1$ \\
\hline & Normal & 38 (56\%) & 11 (48\%) & $8(50 \%)$ & $5(63 \%)$ & $2(67 \%)$ & 1 (100\%) & $1(100 \%)$ \\
\hline & High & $30(44 \%)$ & $\begin{array}{l}12(52 \%) \\
p=0.629\end{array}$ & $8(50 \%)$ & $3(37 \%)$ & $1(33 \%)$ & - & - \\
\hline \multirow[t]{3}{*}{$C D-R$} & & $n=66$ & $n=20$ & $n=13$ & $n=2$ & $\mathrm{n}=4$ & - & - \\
\hline & Normal & $56(85 \%)$ & $12(60 \%)^{*}$ & $10(77 \%)$ & 2 (100\%) & $1(25 \%)$ & - & - \\
\hline & High & $10(15 \%)$ & $\begin{array}{l}8(40 \%)^{*} \\
p=0.027\end{array}$ & $3(23 \%)$ & - & $3(75 \%)$ & - & - \\
\hline \multirow{3}{*}{ CD-NR } & & $\mathrm{n}=38$ & $n=6$ & $\mathrm{n}=5$ & - & - & - & $n=1$ \\
\hline & Normal & $36(95 \%)$ & $6(100 \%)$ & $5(100 \%)$ & - & - & - & 1 (100\%) \\
\hline & High & $2(5 \%)$ & - & - & - & - & - & - \\
\hline
\end{tabular}

WT, wild-type (without CARD15 mutation); 3020insC, R702W, and G908R, mutations of the CARD15 gene (homozygous plus heterozygous); PI, permeability index; $C D$, Crohn's disease; CD-R, first degree relative of a patient with $C D ; C D-N R$, non-related household member living with a patient with $C D$. *Significantly different incidence of high and normal permeability between wild-type and mutation subgroups.

Normal permeability, $\mathrm{PI} \leqslant 0.03$; high permeability, $\mathrm{PI}>0.03 ; \chi^{2}$ test paired comparison (Fisher's exact).

CD-NR groups had no significant effect on intestinal permeability values (data not shown).

Intestinal permeability did not differ between CD-R subgroups. CD-R household members were living in the same household with patients at the time of diagnosis and at the time of the test; CD-R non-household members were living on their own during the whole period.

\section{Genetic analysis}

Three major polymorphisms within the coding region of the CARD15 gene (3020insC, R702W, and G908R) have been associated with $\mathrm{CD}$. The results of the present study are illustrated in table 2. A significantly higher prevalence of the frameshift mutation 3020insC was found in CD patients and their first degree relatives compared with controls. The proportion of the heterozygous plus homozygous mutations was $22 \%$, being similar to that found in the CD-R group $(23 \%)$. In the CD-NR group, the corresponding value was $12 \%$. A similar tendency was obvious for the R702W

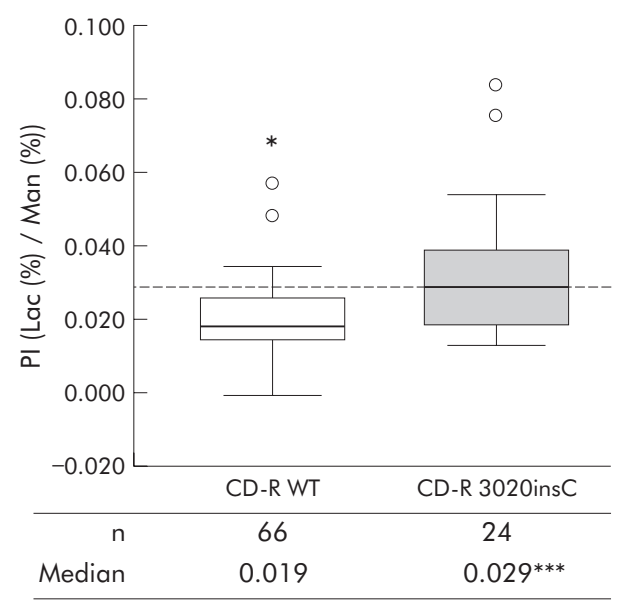

Figure 2 Intestinal permeability in Crohn's disease first degree relatives without a CARD 15 mutation (CD-R WT) and with 3020 ins C or 3020insC-R702W mutations (CD-R 3020insC). Intestinal permeability was significantly increased in CD-R with mutations compared with $C D-R$ without mutations. PI, permeability index (lactulose (Lac)/mannitol (Man) ratio). ${ }^{* * *} p=0.001$ between the CD-R WT and CD-R 3020insC groups (Mann-Whitney test). Broken line indicates the upper limit of normal permeability. Data are presented as box plats. mutation while data for the G908R did not differ between the study groups (table 2 ).

\section{Association between mucosal permeability and CARD 15 mutations}

Looking for a possible link between the prevalence of the CARD15 mutation and intestinal barrier function, individual study groups were divided into subgroups according to the occurrence of a mutation (table 3). CD patients without (wild-types) and with at least one CARD15 mutation (mutants) did not differ with respect to mucosal permeability. In CD wild-types and patients with the 3020insC mutation, the frequencies of elevated permeability were $44 \%$ and $52 \%$, respectively. In contrast, in healthy CD first degree relatives we found a significant association between CARD15 3020insC mutation and intestinal permeability (table 3). Only $15 \%$ of the wild-types, but $40 \%$ of 3020 ins $\mathrm{C}$ mutation carriers had increased values. In addition, median values for intestinal permeability were significantly higher in CD-R with the 3020ins C mutation compared with CD-R wild-types (fig 2). In four CD-R, combined mutations (3020insC and $\mathrm{R} 702 \mathrm{~W}$ ) occurred; three of them also had increased permeability. In contrast, neither R702W nor G908R alone showed a clear association. In CD-NR, all of the mutation carriers had normal permeability (table 3 ).

\section{DISCUSSION}

In the present study there were three main findings. Firstly, healthy first degree relatives of patients with $C D$ showed increased intestinal permeability in contrast with unrelated household members and controls. Secondly, the prevalence of the CARD15 3020insC mutation was similar in first degree relatives and CD patients and higher compared with controls. Thirdly, in healthy first degree relatives, high mucosal permeability and the presence of a CARD15 3020insC mutation were significantly associated.

Based on a large sample size compared with other studies, ${ }^{1}{ }^{46-8}$ our analysis clearly revealed intestinal permeability defects in $26 \%(34 / 129)$ of healthy first degree CD relatives. Furthermore, there was no significant difference between those living in the same household with a CD patient since the time of diagnosis and those living on their own. Thus the parameter "sharing an environment" obviously does not explain the different permeability patterns in $\mathrm{CD}$ first degree relatives. Changes in permeability seem to reflect genetic rather than environmental factors. This is in line with the results in the group of non-blood relatives. All shared a household with a patient but only $6 \%(4 / 66)$ had a 
PI above the normal level. However, data in the literature appear contradictory. Some studies documented high baseline permeability not only in related ${ }^{3}{ }^{6-8}$ but also in unrelated household members (spouses) of CD patients. ${ }^{712}$ These reports were partly limited by sample size and some used a lower "upper limit of normality" for permeability values than those used in our study (for example, $0.0195^{8}$ and $0.0170^{7}$ ), which may have had a slight effect on the frequency distribution of the data. However, under close scrutiny, they also revealed clear evidence that epithelial hyperpermeability of the intestine, in response to insults causing barrier dysfunction, was restricted to $\mathrm{CD}$ first degree relatives and was not seen in spouses. ${ }^{8}$ In summary, data indicate a genetic rather than an environmental background for intestinal barrier dysfunction in CD.

Our results add further support to the role and value of intestinal barrier function as a subclinical marker in families with CD. The question of whether increased intestinal permeability may be a primary or causative factor in disease development cannot be answered from the present data. It is assumed by some authors that slight inflammation may be a primary event preceding barrier disruption. A recent study using faecal calprotectin concentration for detection of intestinal inflammation reported $88 \%(n=43)$ of $C D$ patients, $49 \%(\mathrm{n}=74)$ of CD first degree relatives, and $13 \%$ $(n=2)$ of $C D$ spouses with abnormally high values. ${ }^{24}$ The authors proposed a genetic basis for subclinical inflammation representing a risk factor for $\mathrm{CD}$. Others reported a relationship between faecal calprotectin and intestinal permeability. ${ }^{25}$ It could be that subliminal inflammation may precede barrier disruption. However, a definition of causality is difficult in this case because both factors present at the same time as secondary factors, perpetuating and worsening the processes in the intestinal mucosa. In our opinion neither method (faecal calprotectin detection nor in vivo permeability measurement) provides sufficient sensitivity to answer this question. Analysis of mucosal tight junction components and metabolisms in CD first degree relatives, for example, may be more useful. However, the results of Thjodleifsson and colleagues $^{24}$ stress the importance of a genetic basis of CD and support the role of subclinical markers.

A possible genetic mechanism involved in barrier dysfunction in healthy family members is unknown. An interesting candidate for such a genetic factor is the CARD15 gene, or rather its mutations associated with CD. In accordance with the main findings in European, Canadian, and US Caucasian populations, ${ }^{14-18} 26$ our gene analysis revealed a significantly higher prevalence of the CARD15 3020insC mutation in CD patients compared with controls. Interestingly, this was also true for CD first degree relatives. Values were similar to those of patients and, in contrast with non-blood relatives, significantly higher compared with controls. Obviously, there was transmission of the polymorphisms within genetically related persons, which supports recent data. ${ }^{27}$ However, the striking finding of the present study was that healthy first degree relatives carrying a 3020insC mutation were more often characterised by high intestinal permeability than wildtypes. Eighty five per cent of CD-R wild-types had normal permeability.

A relationship between CD and the CARD15 mutation is assumed. However, CARD15 mutations are not generally present in all CD patients, and are rare in some populations (for example, they were not found in Japanese or Chinese Han populations ${ }^{28}{ }^{29}$ or in an Icelandic population, as seen in the study of Thjodleifsson and colleagues ${ }^{24}$ ). Thus CARD15 mutations may be one risk factor for CD development but not the sole factor. Interestingly, we and others found that 3020insC was associated with a special phenotype of CD, with younger age at diagnosis, ileal involvement, ileocaecal resections, and a high risk of postoperative relapse and reoperation $^{161719}$ 3020insC may therefore characterise a subgroup or subtype of $\mathrm{CD}$ associated mainly with ileitis. Regarding a possible link with intestinal barrier function, the physiological consequences of a CARD15 mutation are crucial. As already mentioned, the CARD15/NOD2 protein is an intracellular receptor for muramyl dipeptide, a conserved structure in bacterial peptidoglycan. ${ }^{20}{ }^{21}$ In human intestinal tissue the protein is expressed predominantly in Paneth cells in the crypts of the terminal ileum. ${ }^{30}$ Clear functional consequences have been demonstrated, mainly for the frameshift mutation 3020insC. 3020insC results in diminished bacterial recognition and hyporesponsiveness to bacterial muramyl dipeptide, showing attenuated or complete loss of NFKB activation. A signalling defect of the innate immunity was indicated..$^{18} 202231$ Studies in mice ${ }^{31}$ and humans $^{32}$ reported a 3020insC associated diminished expression of antimicrobial peptides such as defensins in intestinal Paneth cells. The authors speculated that defensin deficiency may lead to impaired mucosal barrier and susceptibility to bacterial invasion which could trigger inflammation and loss of tolerance against the luminal flora. ${ }^{32}$ A direct effect of bacterial products on tight junction integrity ${ }^{33}$ or a secondarily exaggerated local inflammatory response of the adaptive immune system (that is, high TNF- $\alpha$ or NFKB production) ${ }^{13}$ may cause or facilitate barrier break. Both of these pathways are possible and may play a role. They might gain special importance, representing an early event, probably pointing towards a risk in a subgroup of persons. Interestingly, an older study reported abnormal faecal flora in patients with $\mathrm{CD}$ and their first degree relatives. ${ }^{34}$

However, if there is an association between the CARD15 mutation and impaired barrier function, why is this association not obvious in CD patients but only in a subgroup of healthy first degree relatives of CD patients? These are two completely different situations. One is the healthy first degree relative of a CD patient. This person has no clinical symptoms of CD but has a CARD15 mutation and impaired barrier function which may bear a risk but which may not develop any clinical relevance. The other situation is a confirmed diagnosis of a chronic inflammatory bowel disease (that is, CD). In our study, $44 \%$ of $C D$ patients without any CARD 15 mutation (that is, wild-types) showed pathologically high permeability values. In current $C D$, various factors, including the dynamism of the inflamed disturbed barrier itself, influence barrier function. Early events could be easily masked. These factors are mainly drugs such as prednisolone or mesalamine, ${ }^{35}$ or the effects of mal- and dysnutrition, ${ }^{36}{ }^{37}$ stress, $^{38}{ }^{39}$ or changes in luminal bacterial load and composition, ${ }^{34} 40$ which are all associated with $\mathrm{CD}$ and which are accompanied by reinforcement or impairment of the intestinal barrier.

In summary, the present data indicate a genetic rather than an environmental basis for the intestinal barrier dysfunction in CD. From our data, we speculate that the CARD15 3020insC mutation could be one genetic factor involved in impairment of intestinal barrier function. However, it is obvious that this is not the only factor. Association between the CARD15 gene mutation and intestinal hyperpermeability in healthy first degree CD relatives may be one step towards the identification of other target genes involved in similar processes or possibly interacting with the CARD15 gene, as recently discussed ${ }^{42} 43$ and proposed for the $D L G 5$ gene. ${ }^{44}$ Our data point towards a very early step in the disease process. Considering the high intestinal bacterial load in CD patients compared with controls, ${ }^{41}$ early barrier dysfunction gains special significance with respect to the pathogenesis of CD. However, longitudinal studies in CD families are necessary to investigate which additional factors may lead to the outbreak of the disease. 


\section{ACKNOWLEDGEMENTS}

We thank Dr H Radtke (Department of Transfusion Medicine, Charité Campus Mitte, Charité-Universitätsmedizin Berlin) for support in recruiting healthy volunteers.

We thank Martina Werich and Bettina Bochow for excellent technical assistance and Sheila Fryk for correcting the English. This study was supported by a grant from the German Ministry for Education and Research (BMBF)-Competence Network "Inflammatory Bowel Disease": 01GI0284 TP 1.17.

\section{Authors' affiliations}

S Buhner, C Buning, J Genschel, K Kling, D Herrmann, S Krueger, H H-J

Schmidt, $\mathbf{H}$ Lochs, Department of Gastroenterology, Hepatology, and

Endocrinology, Campus Charité Mitte, Charité-Universitätsmedizin

Berlin, Germany

A Dignass, Department of Hepatology and Gastroenterology, Campus Virchow Klinikum, Charité-Universitätsmedizin Berlin, Germany

I Kuechler, Institute of Medical Biometry, Campus Charité Mitte, CharitéUniversitätsmedizin Berlin, Germany

Conflict of interest: None declared.

\section{REFERENCES}

1 Katz KD, Hollander D, Vadheim CM, et al. Intestinal permeability in patients with Crohn's disease and their healthy relatives. Gastroenterology 1989;97:927-31

2 Teahon K, Smethurst P, Levi AJ, et al. Intestinal permeability in patients with Crohn's disease and their first degree relatives. Gut 1992;33:320-3.

3 Hollander D. Permeability in Crohn's disease: altered barrier functions in healthy relatives? Gastroenterology 1993;104:1848-51.

4 May GR, Sutherland LR, Meddings JB. Is small intestinal permeability really increased in relatives of patients with Crohn's disease? Gastroenterology 1993; 104:1627-32.

5 Wyatt J, Vogelsang $\mathrm{H}$, Hubl W, et al. Intestinal permeability and the prediction of relapse in Crohn's disease. Lancet 1993:341:1437-9.

6 Yacyshyn BR, Meddings JB. CD45RO expression on circulating CD19+ B cells in Crohn's disease correlates with intestinal permeability. Gastroenterology 1995; 108:132-7.

7 Peeters M, Geypens B, Claus D, et al. Clustering of increased small intestinal permeability in families with Crohn's disease. Gastroenterology $1997 ; 113: 802-7$

8 Soderholm JD, Olaison G, Lindberg E, et al. Different intestinal permeability patterns in relatives and spouses of patients with Crohn's disease: an inherited defect in mucosal defence? Gut 1999:44:96-100.

9 Podolsky DK. Inflammatory bowel disease. N Engl J Med 2002;347:417-29.

10 DeMeo MT, Mutlu EA, Keshavarzian A, et al. Intestinal permeation and gastrointestinal disease. J Clin Gastroenterol 2002;34:385-96.

11 Irvine EJ, Marshall JK. Increased intestinal permeability precedes the onset of Crohn's disease in a subject with familial risk. Gastroenterology 2000; 119:1740-4

12 Breslin NP, Nash C, Hilsden RJ, et al. Intestinal permeability is increased in a proportion of spouses of patients with Crohn's disease. Am J Gastroenterol 2001;96:2934-8.

13 Ogura $Y$, Bonen DK, Inohara N, et al. A frameshift mutation in NOD2 associated with susceptibility to Crohn's disease. Nature 2001;411:603-6.

14 Hugot JP, Chamaillard M, Zouali $\mathrm{H}$, et al. Association of NOD2 leucine-rich repeat variants with susceptibility to Crohn's disease. Nature 2001:411:599-603.

15 Hampe J, Cuthbert A, Croucher PJ, et al. Association between insertion mutation in NOD2 gene and Crohn's disease in German and British populations. Lancet 2001;357:1925-8.

16 Cuthbert AP, Fisher SA, Mirza MM, et al. The contribution of NOD2 gene mutations to the risk and site of disease in inflammatory bowel disease. Gastroenterology 2002;122:867-74.

17 Vermeire S, Wild G, Kocher K, et al. CARD1 5 genetic variation in a Quebec population: prevalence, genotype-phenotype relationship, and haplotype structure. Am J Hum Genet 2002;71:74-83.
18 Bonen DK, Ogura Y, Nicolae DL, et al. Crohn's disease-associated NOD2 variants share a signaling defect in response to lipopolysaccharide and peptidoglycan. Gastroenterology 2003;124:140-6.

19 Buning C Genschel J, Buhner S, et al. Mutations in the NOD2/CARD15 gene in Crohn's disease are associated with ileocecal resection and are a risk factor for reoperation. Aliment Pharmacol Ther 2004;19:1073-8.

20 Inohara N, Ogura Y, Fontalba A, et al. Host recognition of bacterial muramyl dipeptide mediated through NOD2. Implications for Crohn's disease. J Biol Chem 2003;278:5509-12.

21 Girardin SE, Boneca IG, Viala J, et al. Nod2 is a general sensor of peptidoglycan through muramyl dipeptide (MDP) detection. J Biol Chem 2003;278:8869-72.

22 Hisamatsu T, Suzuki M, Reinecker HC, et al. CARD15/NOD2 functions as an antibacterial factor in human intestinal epithelial cells. Gastroenterology 2003; 124:993-1000

23 Buhner S, Reese I, Kuehl F, et al. Pseudoallergic reactions in chronic urticaria are associated with altered gastroduodenal permeability. Allergy 2004:59:1118-23.

24 Thjodleifsson B, Sigthorsson G, Cariglia N, et al. Subclinical intestinal inflammation: an inherited abnormality in Crohn's disease relatives? Gastroenterology 2003;124:1728-37.

25 Berstad A, Arslan G, Folvik G. Relationship between intestinal permeability and calprotectin concentration in gut lavage fluid. Scand J Gastroenterol 2000;35:64-9.

26 Giachino D, van Duist MM, Regazzoni S, et al. Analysis of the CARD15 variants R702W, G908R and L1007fs in Italian IBD patients. Eur J Hum Genet 2004; 12:206-12

27 Esters N, Pierik M, van Steen K, et al. Transmission of CARD15 (NOD2) variants within families of patients with inflammatory bowel disease. Am J Gastroenterol 2004;99:299-305.

28 Guo QS, Xia B, Jiang Y, et al. NOD2 3020insC frameshift mutation is not associated with inflammatory bowel disease in Chinese patients of Han nationality. World J Gastroenterol 2004;10:1069-71.

29 Yamazaki K, Takazoe M, Tanaka T, et al. Absence of mutation in the NOD2/ CARD 15 gene among 483 Japanese patients with Crohn's disease. J Hum Genet 2002;47:469-72.

30 Lala S, Ogura Y, Osborne C, et al. Crohn's disease and the NOD2 gene: a role for Paneth cells. Gastroenterology 2003;125:47-57.

31 Kobayashi KS, Chamaillard M, Ogura Y, et al. Nod2-dependent regulation of innate and adaptive immunity in the intestinal tract. Science 2005;307:731-4.

32 Wehkamp J, Harder J, Weichenthal M, et al. NOD2 (CARD15) mutations in Crohn's disease are associated with diminished mucosal alpha-defensin expression. Gut 2004;53:1658-64.

33 Sakaguchi T, Kohler H, Gu X, et al. Shigella flexneri regulates tight junctionassociated proteins in human intestinal epithelial cells. Cell Microbiol 2002:4:367-81.

34 Van de Merwe JP, Schroder AM, Wensinck F, et al. The obligate anaerobic faecal flora of patients with Crohn's disease and their first-degree relatives. Scand J Gastroenterol 1988;23:1125-31.

35 Miehsler W, Puspok A, Oberhuber T, et al. Impact of different therapeutic regimens on the outcome of patients with Crohn's disease of the upper gastrointestinal tract. Inflamm Bowel Dis $2001 ; 7: 99-105$

36 De-Souza DA, Greene L. Intestinal permeability and systemic infections in critically ill patients: effect of glutamine. Crit Care Med 2005;33:1 125-35.

37 Ferraris RP, Carey HV. Intestinal transport during fasting and malnutrition. Annu Rev Nutr 2000;20:195-219.

38 Hart A, Kamm MA. Review article: mechanisms of initiation and perpetuation of gut inflammation by stress. Aliment Pharmacol Ther 2002;16:2017-28.

39 Soderholm JD, Yang PC, Ceponis P, et al. Chronic stress induces mast celldependent bacterial adherence and initiates mucosal inflammation in rat intestine. Gastroenterology 2002;123:1099-108.

40 Caradonna L, Amati L, Magrone T, et al. Enteric bacteria, lipopolysaccharides and related cytokines in inflammatory bowel disease: biological and clinical significance. J Endotoxin Res 2000;6:205-14.

41 Swidsinski A, Ladhoff A, Pernthaler A, et al. Mucosal flora in inflammatory bowel disease. Gastroenterology 2002;122:44-54.

42 Negoro K, McGovern DP, Kinouchi Y, et al. Analysis of the IBD5 locus and potential gene-gene interactions in Crohn's disease. Gut 2003;52:541-6.

43 van Heel DA, Dechairo BM, Dawson G, et al. The IBD6 Crohn's disease locus demonstrates complex interactions with CARD 15 and IBD5 disease-associated variants. Hum Mol Genet 2003;12:2569-75.

44 Stoll M, Corneliussen B, Costello CM, et al. Genetic variation in DLG5 is associated with inflammatory bowel disease. Nat Genet 2004;36:476-80. 